\title{
Rendering Softness: Integration of Kinesthetic and Cutaneous Information in a Haptic Device
}

\author{
Enzo Pasquale Scilingo, Matteo Bianchi, Giorgio Grioli, and Antonio Bicchi, Fellow, IEEE
}

\begin{abstract}
While it is known that softness discrimination relies on both kinesthetic and cutaneous information, relatively little work has been done on the realization of haptic devices replicating the two cues in an integrated and effective way. In this paper, we first discuss the ambiguities that arise in unimodal touch, and provide a simple intuitive explanation in terms of basic contact mechanics. With this as a motivation, we discuss the implementation and control of an integrated device, where a conventional kinesthetic haptic display is combined with a cutaneous softness display. We investigate the effectiveness of the integrated display via a number of psychophysical tests and compare the subjective perception of softness with that obtained by direct touch on physical objects. Results show that the subjects interacting with the integrated haptic display are able to discriminate softness better than with either a purely kinesthetic or a purely cutaneous display.
\end{abstract}

Index Terms-Haptic interfaces, softness rendering, kinesthesia, cutaneous perception, psychophysics.

\section{INTRODUCTION}

$\mathrm{I}_{\mathrm{s}}^{\mathrm{N}}$ $\mathrm{N}$ the effort to create a feeling of real presence in a virtual or remote environment, we must give precedence to touch over all other senses because its perceptions have the most compelling character of reality [1]. For touch to be real, we need to design haptic devices capable of reproducing each dimension of tactile sensation. When an object is haptically explored, information about texture, hardness, weight, shape, size, and thermal properties are conveyed. Among these dimensions, the material properties of hardness and texture appear to be of particular importance. According to Lederman and Klatzky [2], hardness, together with texture and thermal conductivity, is the maximally available dimension for processing after initial contact because, as opposed to geometric properties, they do not need to be coded with reference to a coordinate system. Indeed, it has been observed that material properties provide faster perceptual access than the geometric properties of size and shape [3]. Further behavioral [4], [5] and neuroimaging [6] studies found out that, while remembering or imagining geometric features of an object evokes visual imagery, the interrogation of material features evokes the processing of semantic object representations. This suggests that these are fundamentally tactile-related properties, not easily accessed via vision or visual imagery.

The two modalities of haptics, kinesthesia, and cutaneous information, are involved to different extents in the tactile perception of different properties: for instance, while weight is dominated by kinesthesia, thermal sensations are purely cutaneous. However, perception and precise discrimination of softness depend on both cutaneous and kinesthetic

- The authors are with the Interdepartmental Research Center "E. Piaggio," University of Pisa, via Diotisalvi 2, 56126 Pisa, Italy. E-mail: \{e.scilingo, matteo.bianchi, giorgio.grioli, bicchi\}@centropiaggio.unipi.it.

Manuscript received 13 July 2009; revised 23 Nov. 2009; accepted 3 Jan. 2010; published online 13 Jan. 2010.

Recommended for acceptance by H. Noma.

For information on obtaining reprints of this article, please send e-mail to: toh@computer.org, and reference IEEECS Log Number TH-2009-07-0048. Digital Object Identifier no. 10.1109/ToH.2010.2.

$1939-1412 / 10 / \$ 26.00$ (C) 2010 IEEE information: psychophysical experiments leading to this conclusion have been reported in the literature, starting with the well-known 1995 paper by Srinivasan and LaMotte [7].

This notwithstanding, the technology of haptic devices is still today unsatisfactory as far as softness rendering is concerned. Although purely kinesthetic devices, such as, e.g., the PHANToM [8] or the Delta Haptic Device (DHD) by Force Dimension [9], have achieved outstanding results in displaying hardness within their impedance range (or $Z$ width), the impossibility to provide cutaneous cues severely limits their capabilities.

On the other hand, although recent advancements in cutaneous displays [10], [11], [12] have provided promising results, a technical difficulty remains to achieve sufficient resolution of the stimuli so as to convey a convincing softness information. Devices specifically intended to display softness properties have been proposed in the literature (see, e.g., [13], [14], [15]), which are based on surrogating detailed contact shape information with information on the contact area on the fingertip and its changes with varying contact force. These displays have proved able to evoke a reliable softness sensation, enabling better discrimination than a similar, but purely kinesthetic display [13] for objects in a given class. The main limitations of this display were its limited workspace and softness range. Moreover, the device was unable to decouple the rendering of cutaneous and kinesthetic information.

To fully exploit the integrated nature of human tactile perception and correspondingly enlarge the class of objects that can be discriminated, in this paper we propose a combination of kinesthetic and contact area displays, so as to achieve independent and accurate rendering of both kinesthetic and cutaneous cues. To motivate the need for integrated displays, we consider a simplified example involving the mechanics of contact between elastic bodies, illustrating "unimodally ambiguous" objects, i.e., objects that provide equal kinesthetic but different cutaneous cues, or the other way around.

We then describe an integrated display, and the control technique to achieve independent control of the kinesthetic 


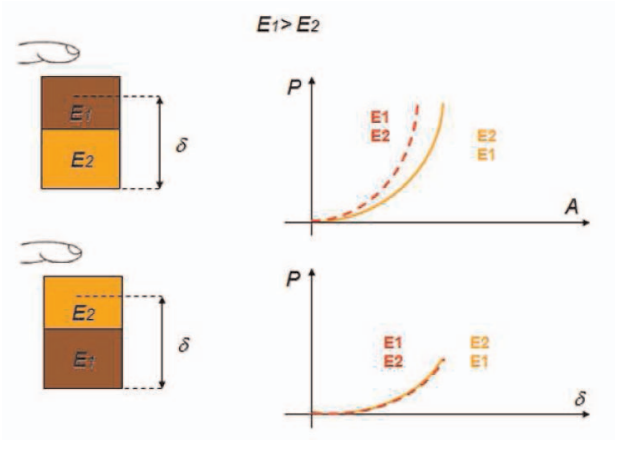

Fig. 1. An object comprised of two layers of different materials is probed for softness from the two sides. The resultant force-displacement $(P / \delta)$ curve is equal, while the force-area $(P / A)$ curve is steeper when the stiffer layer is above.

and cutaneous information. To assess the performance of the integrated display, we conduct psychophysical experiments and compare the subjective perception of softness with that obtained by direct touch on physical objects. Results show that the subjects interacting with the integrated haptic display are able to discriminate softness better than with either a purely kinesthetic or a purely cutaneous display.

\section{Motivation: Ambiguities in Unimodal Touch}

When a fingertip (or other tactually endowed parts of the body) enters in contact with an object, a complex mechanical interaction occurs, which generates tactile stimuli for the various receptors in the skin and in the proprioceptive system. These mechanisms are very complex and not completely understood as yet [16]. While it is beyond the scope of this paper to account for these phenomena in detail, it will suffice for our purposes to provide a conceptual model of how softness information can be elicited from raw sensor data, which is abstract enough as to allow a tractable, yet meaningful analysis, and later on a replica by artificial displays.

Let $p(x)$ be the pressure exchanged at point $x$ on the contact surface between two deformable bodies, and let the resultant contact force be denoted by $P$. Clearly, $P$ equals the integral of $p(x)$ over the contact area $A$. Let also $\delta$ denote the overall (rigid) relative displacement between the two bodies, i.e., the relative displacement of two reference frames attached to the two bodies in positions as remote from contact as not to be subject to any deformation. The displacement $\delta$ is set to zero in the relative configuration where the contact is first established.

The resultant force $P$ and rigid displacement $\delta$ are simple and very useful "abstractions" of contact mechanics. Indeed, observing, e.g., the process of tactile probing for softness discrimination, the relationship between their evolutions provides a direct and very relevant information on softness-analogous to the macroscopic force-displacement curve which characterizes deformability of material samples. Given that sensing resultant forces and kinematic motions are primary objects of kinesthesia, the $P / \delta$ curve of a fingertip/object pair can be considered as a close correlate of kinesthetic information elicited by probing for softness.

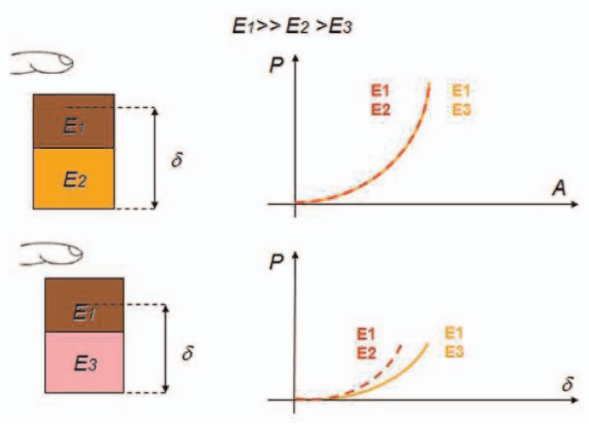

Fig. 2. Two objects producing similar force-area, but different forcedisplacement curves.

To address cutaneous information, a description is needed of the mechanics of contact in the inner part of the fingertip, where mechanoreceptors reside. Contact pressures and displacements on the fingertip surface generate a distribution of stress and strain tensors in the dishomogeneous, inelastic material whose accurate modeling is very difficult. However, it has been shown in previous work [13] that a considerable part of cutaneous information is retained in the relation between the contact force $P$ and the measure of the region of contact, or contact area $A$. We will, therefore, regard the $P / A$ curve of a fingertip/object pair as a correlate of cutaneous information elicited by probing for softness. ${ }^{1}$

We observe preliminarily that there may exist quite distinct objects which, probed for softness, provide identical kinesthetic information but different cutaneous information; and that the opposite also applies. Indeed, consider objects having a two-layered structure, for which both layers are flat and have similar thickness, but different Young's moduli $E_{1}>E_{2}$. Two objects, each consisting of the superposition of the two layers, but probed from opposite sides, would exhibit the same $P / \delta$, but different $P / A$ (see Fig. 1). The opposite case of two objects offering similar $P / A$, but different $P / \delta$ curves, can also be conceived. Consider three materials for which the Young's moduli are $E_{1}>>E_{2}>E_{3}$, and arrange them in two similar two-layered objects as in (see Fig. 2). A somewhat more detailed illustration of possible unimodal haptic ambiguities can be easily given in terms of the classical Hertzian model of contact. Although this is a very rough model of fingertip contact, it does provide insight in the problem and will inspire and motivate actual psychophysical discrimination experiments. ${ }^{2}$

Consider the simple contact between two elastic bodies, pressed against each other by force $P$. Assume the two bodies are locally spherical at the contact, with radii $R_{1}$ and $R_{2}$, respectively. Let the Young's modulus and Poisson ratio for the material of the two spheres be $E_{1}, \nu_{1}$, resp., $E_{2}, \nu_{2}$. From elementary Hertz contact theory [21], [22], we know that the contact pressure at the contact interface is radially

1. The $P / A$ curve of an object was referred to as its Contact Area Spread Rate (CASR) characteristic in [13].

2. More refined models are available in the biomechanics and haptics literature, such as the modified Hertzian model of [17], [18], the viscoelastic sphere of [19], and the liquid-filled membrane model of [20]. However, these models have only been validated for fingertip contact with rigid objects and neglect the effect of the compliance of the surface of the probed object, which is a crucial aspect of our analysis. 

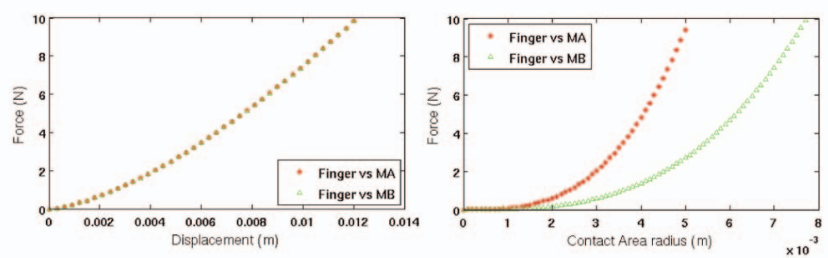

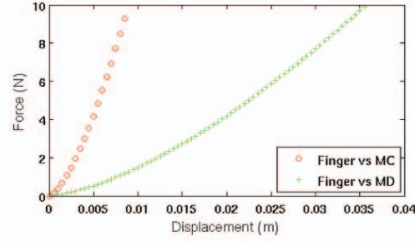

(a)

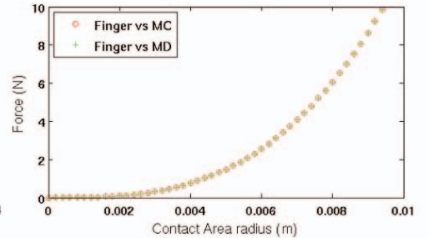

(b)
Fig. 3. Comparison of the (a) force-displacement and (b) force-area curves for finger-object contacts. In the first row, two objects $M_{A}$ and $M_{B}$ have parameters $E_{A}=10^{6} \mathrm{~N} / \mathrm{m}^{2}, R_{A}=2.5 \mathrm{~mm}, E_{B}=1.5 \times 10^{5} \mathrm{~N} / \mathrm{m}^{2}$, $R_{B}=7.3 \mathrm{~mm}$, which satisfy relation (1). In the second row, two objects $M_{C}$ and $M_{D}$ have parameters $E_{C}=2 \times 10^{5} \mathrm{~N} / \mathrm{m}^{2}, R_{C}=30 \mathrm{~mm}$, $E_{D}=2 \times 10^{4} \mathrm{~N} / \mathrm{m}^{2}, R_{D}=3 \mathrm{~mm}$, satisfying relation (2).

symmetric and varies with the distance $r$ from the center of contact as

$$
p=\left(\frac{6 P E^{2}}{\pi^{3} R^{2}}\right)^{\frac{1}{3}}\left(1-\frac{r^{2}}{a^{2}}\right)^{\frac{1}{2}}
$$

where $R^{-1}=R_{1}^{-1}+R_{2}^{-1}$ is the relative radius of curvature,

$$
E=\left(\frac{1-\nu_{1}^{2}}{E_{1}}+\frac{1-\nu_{2}^{2}}{E_{2}}\right)^{-1}
$$

is the equivalent modulus, and $a$ is the radius of the contact area circle, with

$$
a=\left(\frac{3 P R}{4 E}\right)^{\frac{1}{3}} .
$$

From the Hertz model it also follows that the mutual displacement under the same loading condition is

$$
\delta=\left(\frac{9 P^{2}}{16 R E^{2}}\right)^{\frac{1}{3}}
$$

The above equations can be rearranged to obtain a simple relationships between force $P$, displacement $\delta$, and contact area $A=\pi a^{2}$ as

$$
P(\delta)=\frac{4}{3} E \sqrt{R \delta^{3}}
$$

and

$$
P(A)=\frac{4}{3} \frac{E}{R}\left(\frac{A}{\pi}\right)^{\frac{3}{2}} .
$$

These relationships can be used to compare the forcedisplacement $(P / \delta)$ and force-area $(P / A)$ curves obtained for the contact of different pairs of spherical objects.

Consider two contact pairs formed by an idealized (linear elastic spherical) fingertip and two objects $\mathrm{O}_{1}, \mathrm{O}_{2}$. Let the fingertip be characterized by constants $R_{f}, E_{f}$, and $\nu_{f}$. Experimental in vivo characterization of the skin in

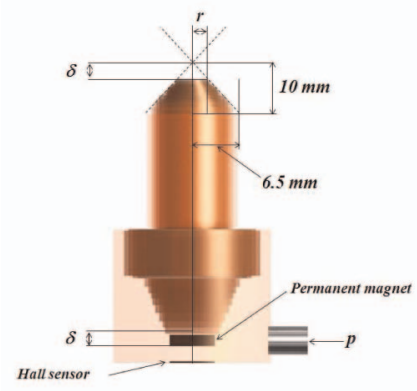

(a)

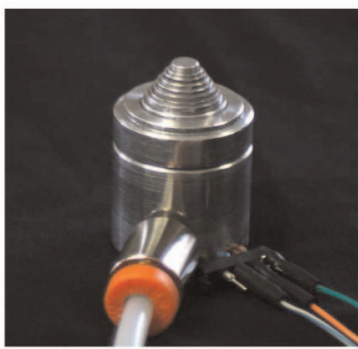

(b)
Fig. 4. (a) Geometry and (b) appearance of the softness display. The prototype has a max. height of $33 \mathrm{~mm}$ and a diameter of $13 \mathrm{~mm}$.

various parts of the body reported in [22] provides values in the range from $0.7 \times 10^{4} \mathrm{~N} / \mathrm{m}^{2}$ to $3.3 \times 10^{4} \mathrm{~N} / \mathrm{m}^{2}$ for the Young's modulus, ${ }^{3}$ whereas a generally accepted value for the Poisson ratio for the skin is 0.5 . We assume $R_{f}=15 \mathrm{~mm}$.

Let $R_{i}, E_{i}, \quad i=1,2$ denote the Young's modulus and radius of the two objects, and $R_{f i}, E_{f i}$ the relative radius and equivalent modulus, respectively. From simple calculations, and assuming for simplicity identical Poisson ratio for objects and fingers, it follows that an object $O_{2}$ would provide the same kinesthetic $(P / \delta)$ information as an object $O_{1}$ if its geometric and elastic parameters satisfy the relation

$$
\frac{1}{R_{2}}=\frac{1}{E_{2}}\left(\frac{E_{f 1}}{R_{f 1}}\right)+\left(\frac{\tilde{E}_{1}}{R_{1}}-\frac{1-\tilde{E}_{1}}{R_{f}}\right),
$$

where $\tilde{E}_{i}=\frac{E_{i}}{E_{i}+E_{f}}$. On the other hand, if

$$
\tilde{R}_{2}=\frac{\tilde{E}_{1}^{2}}{\tilde{E}_{2}^{2}} \tilde{R}_{1},
$$

with $\tilde{R}_{i}=\frac{R_{i}}{R_{i}+R_{f}}$, then the finger would observe the same cutaneous $P / A$ information. From these observations, it follows that if either information is used alone, than there exist simple objects for which their different radii and moduli make them indistinguishable (see Fig. 3).

As a consequence of these results, it is expected that there exist objects whose softness can only be distinguished if both kinesthetic and cutaneous cues are available, and that, in general, tactual discrimination of softness can be improved by an integrated device.

\section{The Integrated Haptic System}

The integrated kinesthetic/cutaneous haptic system is comprised of a softness display combined in series with a commercial haptic interface, the Delta Haptic Device (DHD) (see Fig. 9). The softness display is a pneumatic device consisting of a set of cylinders of different radii. These are assembled in telescopic arrangement (see Fig. 4b), as previously described in [13]. A regulated air pressure is inflated inside acting on the cylinders according to the desired force to be perceived by subjects during indentation. Pressure is applied on all the cylinders. When

3. Parameters in [22] have been obtained by applying the Hertzian model of contact to experimental data from skin indentation with spherical objects. 
the subject finger pushes down against the cylinders, it comes into contact with a surface depending on the height of the cylinders themselves and perceives a resultant force correlated to the pressure. The display can realize a prescribed $P / A$ (CASR) relation [13] (more details are reported in the next section). In order to smooth the change of distribution of pressure against the fingerpad, the cylinders were covered with a latex sleeve. In this way any edge effect was strongly reduced. A proportional Hall sensor placed at the bottom of the inner chamber allows to measure the displacement $\delta$ of the cylinders. A pneumatic servo regulates the chamber pressure $p$ so as to modulate the relation between an external force applied by the user and the contact area.

\subsection{Modeling of the Softness Display}

The softness display can replicate a desired force/area behavior with good accuracy, and it has been demonstrated in the cited literature that subjects had substantially enhanced performance in softness discrimination of objects. However, when used as a stand alone device, the display has limited workspace and softness range. Moreover, it does not allow to implement arbitrary force-area and force-displacement curves independently. The relation between the resultant force $P$ and contact area $A$ in the softness display can be obtained (neglecting losses in the system) by equating the work done by the external force and pressures as

$$
P \Delta \delta=-p \Delta V \Rightarrow P=-p \frac{d V}{d \delta} .
$$

The volume of the inner chamber can be computed geometrically as

$$
V=\pi \frac{R^{2} H}{3}-\pi \frac{\delta^{3} R^{2}}{3 H^{2}},
$$

in which $H$ is the cone height, and $R$ is the external radius at the basis ( $H=10 \mathrm{~mm}$ and $R=6.5 \mathrm{~mm}$ for the prototype at hand). Hence, we have

$$
P=p \pi \frac{\delta^{2} R^{2}}{H^{2}}=p A .
$$

It should be noted here that the tip displacement $\delta$ corresponds to the overall contact displacement, if the softness display is used alone. Hence, to any given $P / A$, the display associates a unique $P / \delta$ profile. This can be changed by coupling the softness display with a purely kinesthetic display, such as the DHD.

The analytical model has been experimentally assessed. The softness display was submitted to indentation tests at different pressures by means of a compressional indentor driven by an electromagnetic actuator. The actuator is a Bruel \& Kjear minishaker, capable of applying a maximum displacement of $10 \mathrm{~mm}$ in the axial direction. The indentor is a metallic cylinder of $1.5 \mathrm{~cm}$ in diameter and $10 \mathrm{~cm}$ in length. The indentor is equipped with a magnetic linear transducer, Vit KD 2,300/6C by KAMAN Science Corporation, and with a load cell sensor, ELH-TC15/100 by Entran, able to detect forces up to $\pm 50 \mathrm{~N}$.

\subsection{Control of the Integrated Display}

In the integrated device, the softness display is connected in series with the DHD. If the axial displacement of the former under load is denoted by $\delta_{s}$, and $\delta_{d}$ is that of the DHD, the overall displacement felt by the probing finger is

$$
\delta=\delta_{s}+\delta_{d}
$$

To replicate two arbitrary kinesthetic and cutaneous curves of a given object marked with the subscript $m$, as given, e.g., by

$$
\left\{\begin{array}{c}
P=\alpha_{m}\left(A_{m}\right) \\
P=\beta_{m}\left(\delta_{m}\right)
\end{array}\right.
$$

the integrated device offers two independent control inputs, the chamber pressure $p$ and the DHD force $P_{d}$. We choose to control the softness display pressure so as to match the force-area curve, i.e., we impose $A=A_{m}$ in (4) and regulate the air pressure as

$$
p=\frac{\alpha_{m}(A)}{A} .
$$

According to the softness display model, a displacement is correspondingly obtained as

$$
\delta_{s}=\frac{H}{R} \sqrt{\frac{A}{\pi}} .
$$

The DHD displacement $\delta_{d}$ is therefore controlled so as to obtain $\delta=\delta_{s}+\delta_{d}=\delta_{m}$. The desired $\delta_{m}$ can be obtained by inversion of the given curve, ${ }^{4}$ i.e., $\delta_{m}=\beta_{m}^{-1}(P)$. Hence,

$$
\delta_{d}=\beta_{m}^{-1}(P)-\frac{H}{R} \sqrt{\frac{P}{p \pi}} .
$$

This value of $\delta_{d}$ is actually used as a reference for a PID control loop for the DHD device, which is fast and stiff enough as to guarantee negligible errors in tracking.

The effectiveness of this control scheme for the integrated display has been experimentally verified by tracking the characteristic curves of different materials. Typical results are reported in Fig. 5, showing good tracking performance for a spherical foam object of radius $R=11 \mathrm{~mm}$ for which the characteristic curves had been experimentally obtained [23] as

$$
\begin{array}{ll}
P=42 \delta_{m}+0.64 & {[\mathrm{~N}],} \\
P=1.4 A_{m}^{2}+0.33 A_{m}+0.554 & {[\mathrm{~N}] .}
\end{array}
$$

To assess the actual quality of the integrated display as a haptic display, however, it is necessary to evaluate how good the haptic rendering of softness is for human subjects interacting with the interface. In the next sections, we describe a series of psychophysical experiments and compare the subjective perception of softness rendered by the display, with that obtained by direct touch of real objects.

\section{Materials and Methods}

\subsection{Subjects}

After written consensus, 15 healthy volunteers participated in the study. Their age ranged from 23 to 30 . None had a history of nerve injury or finger trauma and their finger pads were free of calluses. Five volunteers participated only in the experiments with silicone specimens; five volunteers

4. We assume here the inverse exists, which in practical cases is guaranteed by the monotonicity of the curve. 
(a)
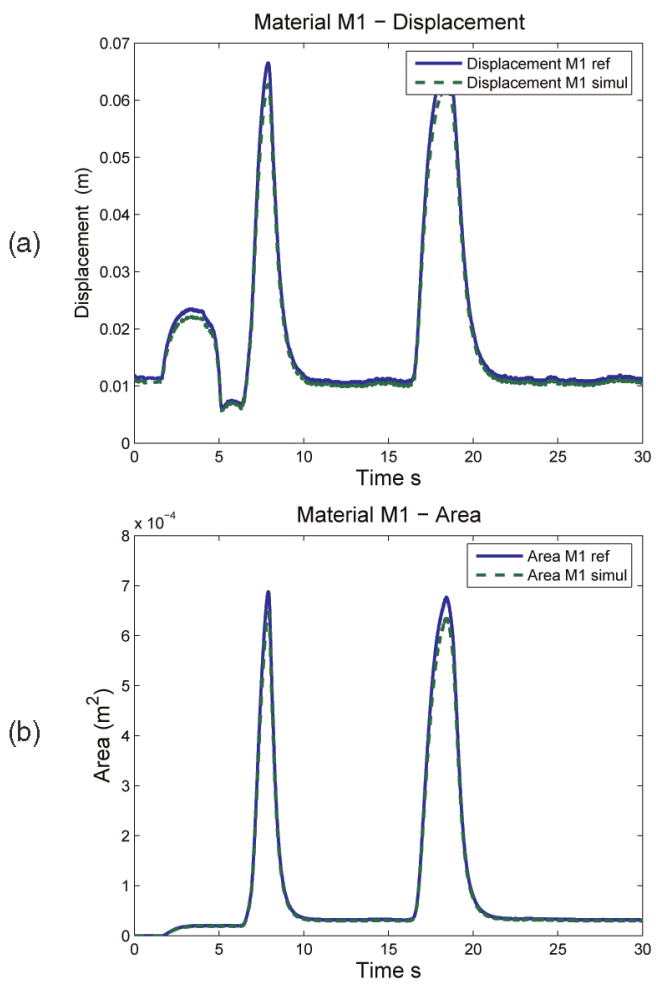

Fig. 5. Experimental results obtained with the integrated display with the (a) independent control of force-displacement and (b) force-area characteristics. Continuous lines represent the response of the reference object to an externally applied force, while dashed lines describe the display outputs.

participated only in the experiments with the haptic display; five volunteers participated in both the experiments. Their handedness was evaluated by the Edinburgh Handedness Inventory (EHI) [24] and they were allowed to use the dominant hand to perform the task. They always performed the tests comfortably sat, blindfolded, and with plugged up ears, to prevent the possible use of any other sensory cues and eliminate any diversion from the task. The chosen arm was locked to the table and the subject was able to move the wrist and fingers only.

\subsection{Physical Specimens}

A set of silicone cylinders was used through one experimental session. Cylinders were made of material obtained by mixing a given quantity of a commercial bicomponent, room temperature-curing silicone (BJB TC-5005A/B), with a variable percentage of plasticizer (BJB TC-5005C), acting as a softener. Varying the amount of softener in the mixture from 0 percent to 45 percent, the Young's modulus decreases as shown in Fig. 6. Each cylinder is $0.7 \mathrm{~cm}$ tall with a radius of $1.85 \mathrm{~cm}$. A set of rigid cylinders having the same radius and height as the previous ones, made of polymethyl methacrylate, was also used.

By suitably combining these cylinders, three classes of specimens were realized, which are described as follows:

1. CL1) Five specimens consisting of two silicone cylinders realized with the same amount of softener, stacked along their axial direction. The used softener percentages were 0 percent, 10 percent, 20 percent, 35 percent, and 45 percent, respectively;

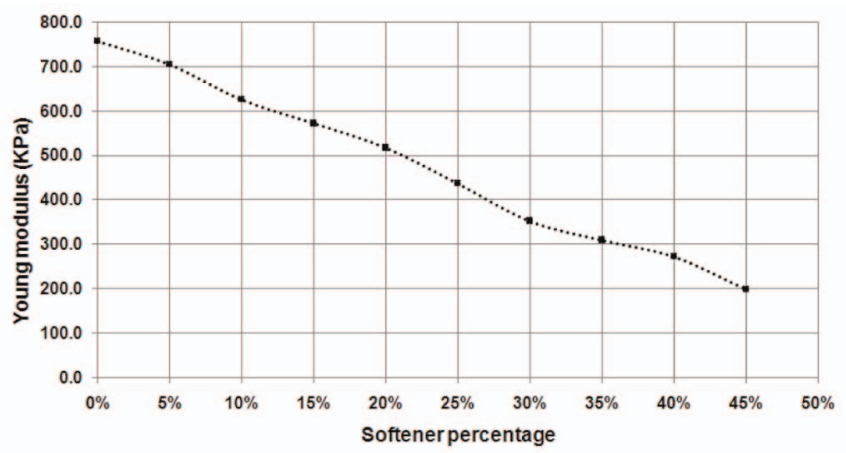

Fig. 6. Relationship between the percentage of softener used in the silicone samples and the Young modulus produced. Measurements used the instrumented indenter described in Section 3.1.

2. CL2) Five specimens consisting of a rigid cylinder placed on the top of a silicone cylinder with the same percentages of softener as in class 1.

3. CL3) Five specimens consisting of two silicone cylinders stacked along their axial direction. The softener percentages of each pair were $(0$ percent45 percent), (10 percent- 35 percent), (20 percent20 percent), (35 percent- 10 percent), (45 percent0 percent). The second softener percentage of each pair refers to the silicone cylinder placed at the bottom of the stacks. These combinations were carefully chosen to have the same overall forcedisplacement characteristic.

\subsection{Rendered Specimens}

Artificial softness specimens were used through the second experimental session, rendering different forcedisplacement, and force-area curves through the haptic display described in previous sections. The display was controlled in three different modes:

1. CM1) the integrated display control method of Section 3.2 is used so as to mimic both the $P / A$ and $P / \delta$ curves of physical specimens in class CL1;

2. CM2) the kinesthetic display (DHD) is controlled to mimic the $P / \delta$ curve of specimens in class CL2, while the softness display is replaced by a rigid surface;

3. CM3) the softness display is controlled to mimic the $P / A$ curve of specimens in class CL3, while the DHD display is used only to compensate for the displacement introduced by the softness display (i.e., to have $\delta_{d}=-\delta_{s}$ in (5)).

\subsection{Design and Procedure}

Experiments were designed to test the ability of subjects to tactually discriminate softness both in normal conditions and through a haptic interface. The first group of experiments, which we will refer to as "direct touch," involved touching physical specimens as described above. The second group, or "rendered touch," involved interaction with artificial specimens rendered through the control of the haptic display. Both groups of experiments included pairwise discrimination and ranking tasks.

Each group of experiments was performed in three different configurations, which were designed to approach three ideal conditions: 


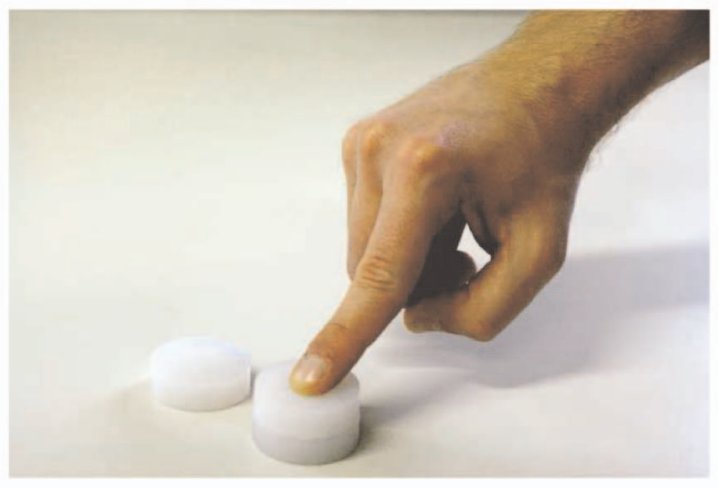

Fig. 7. A subject's hand palpating one of the silicone specimens used in the tests.

1. I) integrated (kinesthetic and cutaneous) tactile information is available to the subject;

2. K) subjects can rely only on kinesthetic information to discriminate the specimens, i.e., cutaneous information is kept unchanged through the experiments;

3. C) subjects can rely only on cutaneous information to discriminate the specimens, i.e., kinesthetic information is kept unchanged through the experiments.

In all tests, subjects had no time limitations and were allowed to check each specimen or haptic stimulus as many times as they wished going back and forth between them at will.

The experiments described in this paper should be compared with the experiments reported by Srinivasan and LaMotte [7], which were also used to investigate the role and relative weight of cutaneous and kinesthetic cues in haptics. First, in [7] no actively controlled haptic interfaces are involved in experiments. Our "direct touch" experiments also differ substantially from those in [7]. Indeed, we do not use anaesthesia to suppress cutaneous cues, rather we construct suitable specimens which equalize the cue itself. Symmetrically, by constructing other specimens which equalize the kinesthetic cue, we avoid the use of "passive" exploration procedures used in [7], whereby the fingertip was rigidly fixed to the tabletop, and specimens were pressed against it. Overall, our techniques are less invasive and allow comparison of human exploration of physical and rendered objects.

\subsection{Experiments on Direct Touch}

Ten subjects participating in this experiment were presented with physical specimens and were asked to judge their softness by touch. They were instructed to do so by pressing vertically or tapping the index or middle finger of their dominant hand against the specimen. Subjects were recommended not to perform movements of the finger across the surface and not to apply lateral forces (see Fig. 7). In this way, according to the literature [2], any anisotropic effect or distortion in softness perception due to the radial/ tangential discrepancy in touch is eliminated, only focusing on normal indentation of the specimens.

Experiments in the integrated condition (I) used specimens in class CL1. Specimens in class CL2 provided for the kinesthetic-only conditions of experiments (K). Indeed, as described above (cf. Fig. 2), these specimens are expected to produce very similar cutaneous cues. Finally, specimens in

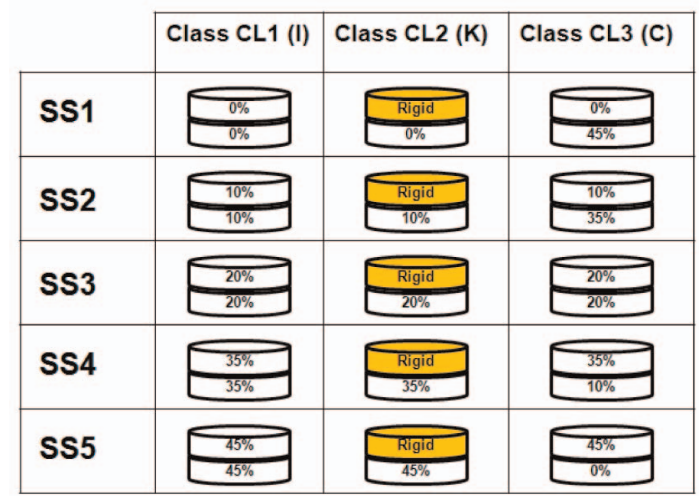

Fig. 8. Naming convention for specimens in different experiments.

class CL3 were used for the cutaneous-only experimental conditions (C) (cf. Fig. 1).

\subsubsection{Pairwise Discrimination}

For each condition (I, K, C), five specimens (denoted by $S S 1$ to $S S 5$ ) in the relative class were used (see Fig. 8).

In each trial, a standard (SS3) and a comparison specimen were presented to the subjects in random order. After probing the specimens, subjects were asked to report which of the two was softer. Each task was performed three times for each condition (I, K, C).

\subsubsection{Ranking}

In the ranking experiment subjects were asked to probe and sort in terms of softness the set of five specimens $S S 1$ to $S S 5$, presented in random order. The specimens in the ranking experiments, for the three different conditions, are as described above (Fig. 8). Ranking tasks were repeated three times for every condition (I, K, C).

\subsection{Experiments on Rendered Touch}

Ten subjects were presented with the integrated haptic display, controlled as to render the softness of different materials, and were asked to judge softness by touch. Subjects were allowed probing and tapping in the vertical direction (corresponding to the axis of the haptic display) and were instructed to avoid exerting lateral forces. A picture of a subject's hand performing the test is shown in Fig. 9.

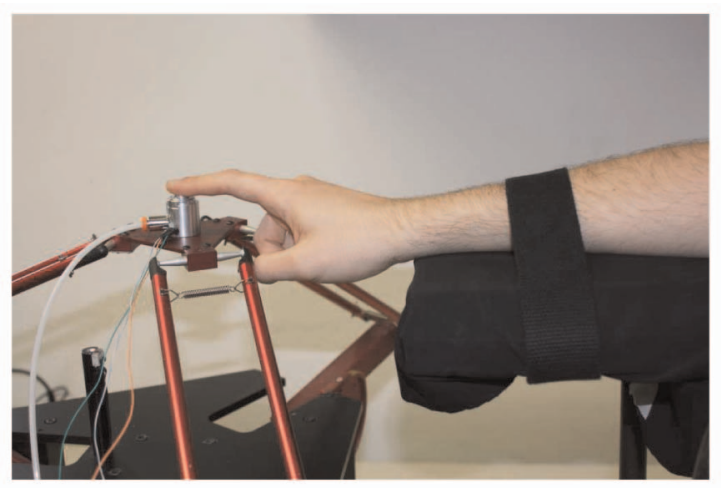

Fig. 9. A subject interacting with the integrated haptic display used in the experiments. 


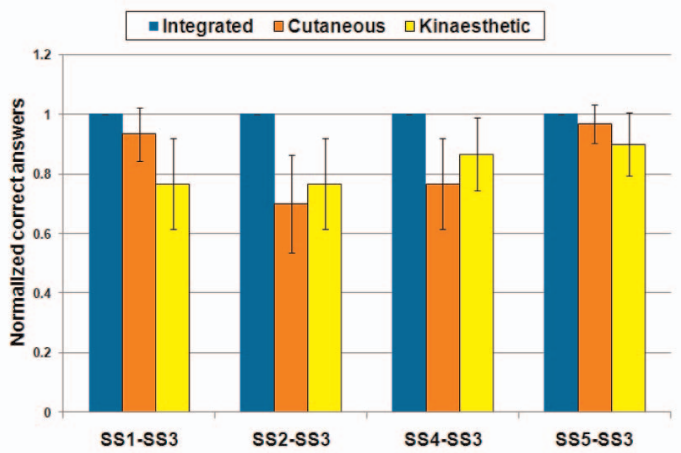

Fig. 10. Results of pairwise discrimination experiments with silicone specimens under three different experimental conditions. Each bar represents the percentage of correct answers for each pair of specimens presented.

\subsubsection{Pairwise Discrimination}

In pairwise discrimination tests, five specimens, denoted as $S H 1-S H 5$ with $S H 3$ as reference, were produced on the haptic system. In the integrated condition (I), specimens were rendered using the control mode CM1; the control mode CM2 was used for condition (K), and mode CM3 for condition (C).

\subsubsection{Ranking}

The same set of five stimuli used in the pairwise discrimination experiment was employed in the ranking experiment. Subjects were presented with new stimuli in less than a second. Subjects were asked to rank these specimens in the three conditions (I, K, C).

\section{EXPerimental RESUlts AND Discussion}

\subsection{Pairwise Discrimination with Physical Specimens}

Results of pairwise discrimination experiments on silicone specimens are reported in Fig. 10. Answers are classified as $X=1$ if the subject correctly identifies the softer specimen, or $X=0$ otherwise. The average number of correct answers $m_{n}$ is represented by the height of the histogram bars in Fig. 10. The statistics of this binary experiment are described by its Bernoulli distribution. Confidence intervals for expected values $E(X)$ with statistical significance $(1-\alpha)$ are also reported in Fig. 10. The intervals are computed as

$$
E(X) \in\left[m_{n}-z_{\frac{\alpha}{2}} \sqrt{\frac{m_{n}\left(1-m_{n}\right)}{N}}, m_{n}+z_{\frac{\alpha}{2}} \sqrt{\frac{m_{n}\left(1-m_{n}\right)}{N}}\right]
$$

with $\alpha=5 \%$, sample size $N=30$, and critical value of the normalized standard distribution $z_{\frac{\alpha}{2}}=1.96$ (from standard statistical tables).

It is worthwhile noting that when subjects can rely on their full tactual ability of manipulation, i.e., in the integrated condition (I), the percentage of correct recognition of the softer specimen in the pair is 100 percent with total confidence for all specimens compared with the standard.

Results obtained when only cutaneous or kinesthetic information were available to subjects $((C)$ and $(K)$, respectively) are clearly weaker than those obtained in integrated conditions, and more so for specimens that are closer to the standard. When stimuli are close to the
TABLE 1

Confusion Matrices of Ranking Experiment Results with Physical Specimens

\begin{tabular}{|c|c|c|c|c|c|c|r|}
\hline CL1 (I) & SS1 & SS2 & SS3 & SS4 & SS5 & N & Relative Accuracy \\
\hline SS1 & 29 & 0 & 1 & 0 & 0 & 30 & $86.67 \%$ \\
\hline SS2 & 0 & 30 & 0 & 0 & 0 & 30 & $100.00 \%$ \\
\hline SS3 & 1 & 0 & 28 & 0 & 1 & 30 & $93.34 \%$ \\
\hline SS4 & 0 & 0 & 0 & 30 & 0 & 30 & $100.00 \%$ \\
\hline SS5 & 0 & 0 & 1 & 0 & 29 & 30 & $96.67 \%$ \\
\hline & & & & & & & Total Accuracy \\
\hline N & 30 & 30 & 30 & 30 & 30 & 150 & $98.67 \%$ \\
\hline
\end{tabular}

\begin{tabular}{|c|c|c|c|c|c|c|r|}
\hline CL2 (K) & SS1 & SS2 & SS3 & SS4 & SS5 & N & Relative Accuracy \\
\hline SS1 & 18 & 3 & 1 & 0 & 8 & 30 & $60.00 \%$ \\
\hline SS2 & 5 & 19 & 1 & 4 & 1 & 30 & $63.34 \%$ \\
\hline SS3 & 0 & 0 & 19 & 9 & 2 & 30 & $43.34 \%$ \\
\hline SS4 & 2 & 7 & 7 & 13 & 1 & 30 & $60.00 \%$ \\
\hline SS5 & 5 & 1 & 2 & 4 & 18 & 30 & $58.00 \%$ \\
\hline & & & & & & & Total Accuracy \\
\hline N & 30 & 30 & 30 & 30 & 30 & 150 & \\
\hline
\end{tabular}

\begin{tabular}{|c|c|c|c|c|c|c|r|}
\hline CL3 (C) & SS1 & SS2 & SS3 & SS4 & SS5 & N & Relative Accuracy \\
\hline SS1 & 24 & 3 & 1 & 1 & 1 & 30 & $80.00 \%$ \\
\hline SS2 & 2 & 23 & 4 & 0 & 1 & 30 & $76.67 \%$ \\
\hline SS3 & 2 & 4 & 16 & 0 & 8 & 30 & $53.34 \%$ \\
\hline SS4 & 1 & 0 & 9 & 18 & 2 & 30 & $60.00 \%$ \\
\hline SS5 & 1 & 0 & 0 & 11 & 18 & 30 & $60.00 \%$ \\
\hline & & & & & & & Total Accuracy \\
\hline N & 30 & 30 & 30 & 30 & 30 & 150 & $66.00 \%$ \\
\hline
\end{tabular}

standard, cutaneous and kinesthetic information are almost equivalent. For stimuli that are farther away from the reference, cutaneous cues appear to provide more information than kinesthetic ones.

\subsection{Ranking of Physical Specimens}

Results from ranking experiments are shown in Table 1, where subjective softness is reported versus objective compliance in a confusion matrix structure for the five specimens, under the three different conditions. Values on the diagonal express the amount of correct answers. The percentage of total accuracy is calculated considering the sum of all correct answers. The integrated modality (I) exhibits a percentage of total accuracy very close to 100 percent. The relative weakness of unimodal cues is apparent also in these experiments. A departure from the perfect ranking is observed in the cutaneous-only condition (C) (66 percent). In kinesthetic-only conditions (K), the discrepancy is even more pronounced (58 percent).

\subsection{Pairwise Discrimination with Rendered Specimens}

Fig. 11 reports results from pairwise discrimination experiments with the haptic display under three different conditions. When the display is controlled to replicate both the kinesthetic and cutaneous cues (integrated mode I), the correct discrimination rate is 100 percent for distant pairs (i.e., $S H 1-S H 3$ and $S H 5-S H 3$ ). As it could be expected, however, for closer pairs (SH4-SH3 and especially $S H 2-S H 3$ ), artificially rendered specimens are discriminated in a considerably poorer way than their physical counterparts.

The performance of subjects in discriminating softness is further lowered if only one cue is rendered. This degradation is more pronounced for rendered kinesthetic cues than for rendered cutaneous cues. In case of $\mathrm{SH} 2-\mathrm{SH} 3$ discrimination, performance of subjects is quite comparable under the three conditions $(\mathrm{I}, \mathrm{K}, \mathrm{C})$, with a percentage of correct recognition close to 80 percent (mean values are $0.75,0.82$, 


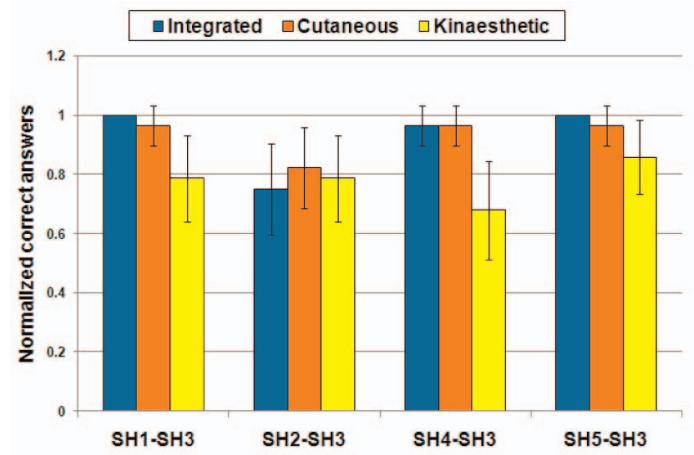

Fig. 11. Results of pairwise discrimination experiments with artificially rendered specimens. Each bar represents the percentage of correct answers for each pair of specimens presented.

and 0.79 for $(\mathrm{I}),(\mathrm{C})$ and $(\mathrm{K})$ conditions with a confidence interval $0.3,0.27$, and 0.29 , respectively). The results support the conclusion that purely kinesthetic haptic displays are inferior in softness discrimination with respect to cutaneous displays used in the experiments, and that integrated displays obtain the best performance, though this is still not quite as good as that of direct touch.

\subsection{Ranking of Rendered Specimens}

Experimental results on ranking experiments of artificially rendered specimens are shown in Table 2. Total accuracy of softness perception using integrated haptic displays is weaker than with physical specimens, yet still strongly correlated to the commanded stimulus (87.35 percent). Results in terms of total accuracy for unimodal cues are very close to the ones observed in ranking experiments with physical specimens. Cutaneous-only stimuli reduce the softness ranking capability to 63.34 percent, while purely kinesthetic rendering further reduces it to 56.67 percent, which is consistent with the results for silicone specimens. We can conclude that the integrated haptic device provides results similar to physical specimens in ranking experiments, and the integrated modality exhibits the highest performance. Indeed, integrating cutaneous cues with kinesthetic cues in artificially rendering haptic softness information increases performance also in tasks such as ranking, which require multiple comparisons and involve haptic memory.

\subsection{Comparison of Direct and Rendered Touch}

The above experimental results of pairwise discrimination and ranking also provide some interesting insight in the evaluation of the performance of our haptic interface through a comparison with direct exploration of physical specimens. Results from the artificial display of kinesthetic cues for softness are comparable with those obtained with real specimens. The effects of naturally and artificially rendered cutaneous cues show that the haptic display provides comparable results than the real case in the middle range of softness. The comparison of results for integrated haptic information between real and artificial stimuli is very satisfactory at both ends of the softness scale. For finer discrimination tasks, i.e., materials that are closer in softness, even the integrated haptic display has obvious limitations. However, it is noteworthy that the total
TABLE 2

Confusion Matrices of Ranking Experiment Results with Rendered Specimens

\begin{tabular}{|c|c|c|c|c|c|c|r|}
\hline CM1 (I) & SH1 & SH2 & SH3 & SH4 & SH5 & N & Relative Accuracy \\
\hline SH1 & 26 & 1 & 1 & 1 & 1 & 30 & $86.67 \%$ \\
\hline SH2 & 1 & 27 & 0 & 1 & 1 & 30 & $90.00 \%$ \\
\hline SH3 & 1 & 0 & 27 & 0 & 2 & 30 & $90.00 \%$ \\
\hline SH4 & 1 & 1 & 1 & 26 & 1 & 30 & $86.67 \%$ \\
\hline SH5 & 1 & 1 & 1 & 2 & 25 & 30 & $83.34 \%$ \\
\hline & & & & & & & Total Accuracy \\
\hline N & 30 & 30 & 30 & 30 & 30 & 150 & $87.34 \%$ \\
\hline
\end{tabular}

\begin{tabular}{|c|c|c|c|c|c|c|r|}
\hline CM2 (K) & SH1 & SH2 & SH3 & SH4 & SH5 & N & Relative Accuracy \\
\hline SH1 & 18 & 4 & 2 & 2 & 4 & 30 & $60.00 \%$ \\
\hline SH2 & 6 & 12 & 6 & 5 & 1 & 30 & $40.00 \%$ \\
\hline SH3 & 1 & 5 & 16 & 7 & 1 & 30 & $53.34 \%$ \\
\hline SH4 & 3 & 6 & 6 & 15 & 0 & 30 & $50.00 \%$ \\
\hline SH5 & 2 & 3 & 0 & 1 & 24 & 30 & $80.00 \%$ \\
\hline & & & & & & & Total Accuracy \\
\hline N & 30 & 30 & 30 & 30 & 30 & 150 & $56.67 \%$ \\
\hline
\end{tabular}

\begin{tabular}{|c|c|c|c|c|c|c|r|}
\hline CM3 (C) & SH1 & SH2 & SH3 & SH4 & SH5 & N & Relative Accuracy \\
\hline SH1 & 21 & 4 & 0 & 2 & 3 & 30 & $70.00 \%$ \\
\hline SH2 & 4 & 23 & 0 & 1 & 2 & 30 & $76.67 \%$ \\
\hline SH3 & 0 & 1 & 19 & 2 & 8 & 30 & $63.34 \%$ \\
\hline SH4 & 5 & 0 & 10 & 15 & 0 & 30 & $50.00 \%$ \\
\hline SH5 & 0 & 2 & 1 & 10 & 17 & 30 & $56.67 \%$ \\
\hline & & & & & & & Total Accuracy \\
\hline N & 30 & 30 & 30 & 30 & 30 & 150 & $63.34 \%$ \\
\hline
\end{tabular}

accuracy of ranking experiments for the three conditions I, $\mathrm{K}$, and $\mathrm{C}$ are comparable for direct and indirect touch.

In summary, results further validate the technology of Contact Area Spread Rate (CASR) cutaneous softness rendering utilized in our haptic interface, and encourage its use in connection with more traditional haptic interfaces.

\section{Conclusion}

In this paper, we have considered the role of kinesthetic and cutaneous information in the discrimination of softness, both in direct and rendered touch. Our results for both cases provide indications consistent with those that Srinivasan and LaMotte [7] described for direct touch using different methods. Relying only on a touch modality limits the possibility for subjects to discriminate softness, while integrating kinesthetic and cutaneous information certainly improves discrimination performance. Two kinds of conclusion can be drawn from this work. Conclusions drawn in Section 5.1 and 5.2, based on physical specimens only, are relevant to cognitive abilities of humans. Conclusions drawn in Section 5.3, 5.4, and 5.5, obtained with artificial specimens, are relevant to performance of haptic displays.

The integrated nature of the somatosensory modality is not reflected in most available haptic displays. As it is observed in [25], the rendering realism of commercial interfaces is severely limited by the lack of cutaneous information. To address such limitation we described a simple and practicable integrated haptic system capable of displaying softness by rendering kinesthetic and cutaneous information. From our preliminary results, what can be noticed is that subjects interacting with this new haptic display actually seem to perceive different degree of softness in a more realistic way. Although preliminary, our results are encouraging toward the realization of convincing integrated kinesthetic and cutaneous displays. 


\section{ACKNOWLEDGMENTS}

This research is partially funded by the EU Commission under contract IST-4-027141 Immersence. The authors gratefully acknowledge the assistance of Nicola Sgambelluri and Giovanni Tonietti in setting up the integrated display controller and the help of Carlo Quartieri in conducting the experiments with subjects. A very special acknowledgment is also extended to Francesca Irene Cavallaro for her assistance in designing the experimental procedure.

\section{REFERENCES}

[1] D. Katz, The World of Touch, L.E. Krueger, ed. Lawrence Erlbaum Assoc., 1989.

[2] S.L. Lederman and R.L. Klatzky, "Relative Availability of Surface and Object Properties During Early Haptic Processing," J. Experimental Psychology: Human Perception and Performance, vol. 23, no. 6, pp. 1680-1707, 1997.

[3] R.L. Klatzky, S.J. Lederman, and C. Reed, "Haptic Integration of Object Properties: Texture, Hardness, and Planar Contour," J. Experimental Psychology: Human Perception and Performance, vol. 15 , no. 1 , pp. $45-57,1989$.

[4] R.L. Klatzky, S.J. Lederman, and D.E. Matula, "Imagined Haptic Exploration in Judgements of Objects Properties," J. Experimental Psychology. Learning, Memory, and Cognition, vol. 17, no. 1, pp. 314322, 1991.

[5] S.J. Lederman and R.L. Klatzky, "Hand Movements: A Window into Haptic Object Recognition," Cognitive Psychology, vol. 19, no. 12, pp. 342-368, 1987.

[6] S.D. Newman, R.L. Klatzky, S.J. Lederman, and M.A. Just, "Imagining Material versus Geometric Properties of Objects: An fMRI Study," Cognitive Brain Research, vol. 23, no. 3, pp. 235-246, 2005.

[7] M.A. Srinivasan and R.H. LaMotte, "Tactile Discrimination of Softness," J. Neurophysiology, vol. 73, no. 1, pp. 88-101, 1995.

[8] http://www.sensable.com, Jan. 2008.

[9] http://www.forcedimension.com, Jan. 2008.

[10] L. Yobas, D.M. Durand, G.G. Skebe, F.J. Lisy, and M.A. Huff, "A Novel Integrable Microvalve for Refreshable Braille Display System," IEEE J. Microelectromechanical System, vol. 12, no. 3, pp. 252-263, June 2003.

[11] V. Levesque, J. Pasquero, V. Hayward, and M. Legault, "Display of Virtual Braille Dots by Lateral Skin Deformation: Feasibility Study," ACM Trans. Applied Perception, vol. 2, no. 2, pp. 132-149, 2005.

[12] S. Koroki, H. Kajimoto, H. Nii, N. Kawakami, and S. Tachi, "Proposal for Tactile Sense Presentation that Combines Electrical and Mechanical Stimulus," Proc. World Haptics Conf., pp. 121-126, 2007.

[13] A. Bicchi, D.E. De Rossi, and E.P. Scilingo, "The Role of the Contact Area Spread Rate in Haptic Discrimination of Softness," IEEE Trans. Robotics and Automation, vol. 16, no. 5, pp. 496-504, Oct. 2000.

[14] K. Fujita and H. Ohmori, "A New Softness Display Interface by Dynamic Fingertip Contact Area Control," Proc. Fifth World Multi Conf. Systemics, Cybernetics and Informatics, pp. 78-82, 2001.

[15] H. Yokota, A. Yamamoto, H. Yamamoto, and T. Higuchi, "Producing Softness Sensation on an Electrostatic Texture Display for Rendering Diverse Tactile Feelings," Proc. Second Joint EuroHaptics Conf. and Symp. Haptic Interfaces for Virtual Environment and Teleoperator Systems, vol. 23, no. 6, pp. 584-585, 2007.

[16] K.O. Johnson, "The Roles and Functions of Cutaneous Mechanoreceptors," Current Opinion in Neurobiology, vol. 11, no. 12, pp. 455-461, 2001.

[17] D. Pawluk and R. Howe, "Dynamic Contact of the Human Fingerpad Against a Flat Surface," J. Biomechanical Eng., vol. 121, no. 6, pp. 605-611, 1999.

[18] D. Pawluk and R. Howe, "Dynamic Lumped Element Response of the Human Fingerpad," J Biomechanical Eng., vol. 121, no. 2, pp. 178-183, 1999.

[19] C. Guler, N. Berme, and S. Simon, "A Viscoelastic Sphere Model for the Representation of Plantar Soft Tissue During Simulations-Kinematics and Kinetics During the Stance Phase of Walking," J. Biomechanics, vol. 31, pp. 847-853, 1998.
[20] E. Serina, E. Mockensturm, C. Mote Jr, and D. Rempel, "A Structural Model of the Forced Compression of the Fingertip Pulp," J. Biomechanics, vol. 31, no. 7, pp. 639-646, 1998.

[21] K.O. Johnson, T. Yoshioka, and F. Vega Bermudez, "Tactile Functions of Mechanoreceptive Afferents Innervating the Hand," J. Clinical Neurophysiology, vol. 17, no. 11, pp. 538-558, 2000.

[22] J. Jachowicz, R. McMullen, and D. Prettypaul, "Indentometric Analysis of In Vivo Skin and Comparison with Artificial Skin Models," Skin Research and Technology, vol. 13, no. 6, pp. 299-309, 2007.

[23] E.P. Scilingo, N. Sgambelluri, G. Tonietti, and A. Bicchi, "Integrating Two Haptic Devices for Performance Enhancement," Proc. World Haptics Conf., pp. 139-144, 2007.

[24] R.C. Oldfield, "The Assessment and Analysis of Handedness: The Edinburgh Inventory," Neuropsychologia, vol. 9, no. 1, pp. 97-113, 1971.

[25] S.J. Lederman and R.L. Klatzky, "Haptic Identification of Common Objects: Effects of Constraining the Manual Exploration Process," Perception \& Psychophysics, vol. 66, no. 4, pp. 618-628, 2004.

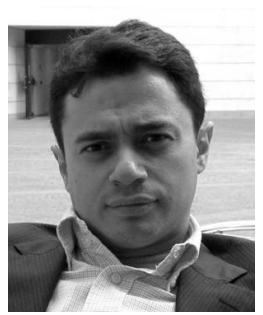

Enzo Pasquale Scilingo received the Laurea degree in electronic engineering from the University of Pisa, Italy, and the PhD degree in bioengineering from the University of Milan, in 1995 and 1998, respectively. He is an assistant professor in electronic and information bioengineering at the University of Pisa. For two years, he was a postdoctoral fellow with the Italian National Research Council and for two years a postdoctoral fellow at Information Engineering Department of the University of Pisa. Currently, he is pursuing his research work mainly at the Interdepartmental Research Center E. Piaggio. His research interests are in haptics, man-machine interface, biomedical and biomechanical signal processing, modeling and control and instrumentation. He is an author of several papers, contributions to international conferences, and chapters in international books.

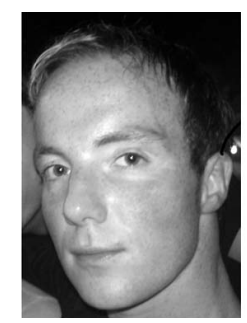

Matteo Bianchi received the bachelors degree cum laude and the masters degree cum laude in biomedical engineering from the University of Pisa, Italy, in 2004 and 2007, respectively. Currently, he is working toward the $\mathrm{PhD}$ degree in automation, robotics, and bioengineering at the Interdepartmental Research Center E. Piaggio of the University of Pisa. His research interests include haptic interfaces, tactile interaction modeling, and human grasping modeling and analysis. He is an authors of contributions to international conferences. 


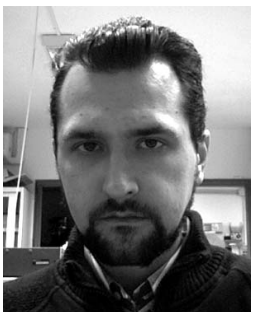

Giorgio Grioli received Laurea specialistica degree in automation engineering from the University of Pisa in 2005. He is currently working toward the $\mathrm{PhD}$ degree in robotics, automation, and bio-engineering in the Department of Electrical Systems and Automation (DSEA) and the Interdepartmental Research Center "E. Piaggio" at the University of Pisa. He won the ICRA best manipulation paper award in 2008. He is an author of some papers on international journals and refereed conferences. His main research interests include human robot interaction, variable impedance actuation, perception in virtual reality, and Haptics.

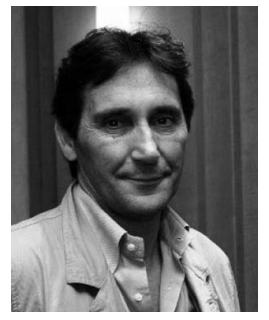

Antonio Bicchi graduated from the University of Bologna in 1988 and was a postdoctoral scholar at M.I.T. A.I. Lab from 1988 to 1990 . He is a professor of system theory and robotics at the University of Pisa. His main research interests include: Dynamics, kinematics and control of complex mechanichal systems, including robots, autonomous vehicles, and automotive systems; haptics and dextrous manipulation; theory and control of nonlinear systems, in particular hybrid (logic/dynamic, symbol/signal) systems. He has published more than 200 papers on international journals, books, and refereed conferences. He currently serves as the director of the Interdepartmental Research Center "E. Piaggio" of the University of Pisa, and as editor in chief of the Conference Editorial Board for the IEEE Robotics and Automation Society (RAS). He is a fellow of the IEEE since 2005. He has served as a vice president of IEEE RAS, a distinguished lecturer, and an editor for several scientific journals including Transactions on Robotics and Automation and Int'l J. Robotics Research. He has organized and cochaired the first WorldHaptics Conference (2005) and Hybrid Systems: Computation and Control (2007).

$\triangleright$ For more information on this or any other computing topic, please visit our Digital Library at www.computer.org/publications/dlib. 